\title{
The Complete Parameters Analysis of the Asymptotic Behaviour of a Logistic Epidemic Model with Two Stochastic Perturbations
}

\author{
Dejun Fan, ${ }^{1,2}$ Ke Wang, ${ }^{2}$ and Ling Hong1 \\ ${ }^{1}$ MOE Key Lab. for Strength and Vibration, Xi'an Jiaotong University, Xi'an, Shaanxi 710049, China \\ ${ }^{2}$ Department of Mathematics, Harbin Institute of Technology (Weihai), Weihai, Shandong 264209, China
}

Correspondence should be addressed to Ling Hong, hongling@mail.xjtu.edu.cn

Received 3 January 2009; Revised 28 July 2009; Accepted 22 August 2009

Recommended by Bin Liu

A simple model of the dynamics of an infectious disease, taking into account environmental variability in the form of Gaussian white noise in the disease transmission rate and the increase in mortality rate due to disease, has been investigated. The probability distribution for the proportion of infected animals, plus its mean, mode, and variance, is found explicitly.

Copyright (C) 2009 Dejun Fan et al. This is an open access article distributed under the Creative Commons Attribution License, which permits unrestricted use, distribution, and reproduction in any medium, provided the original work is properly cited.

\section{Introduction}

In order to describe the dynamics of bovine tuberculosis in possum populations in New Zealand, Roberts and Saha [1] introduced an epidemic model:

$$
\dot{Z}(t)=(p-1) B Z+(\beta C-\alpha)(1-Z) Z,
$$

where $Z(t)$ is the proportion of the population infected with disease against the total population; $p(\in[0,1])$ is the vertical distribution probability; $B$ is the birth rate independent on the total population; $\beta$ is the constant transmission rate; $C$ is the contact rate between individuals; $\alpha$ is the increase of the death rate suffering from infectious disease. It turns out that this model can be used to study other epidemics as well. It is easy to see that (1.1) is a well-known logistic equation with intrinsic growth rate:

$$
r=(p-1) B+\beta C-\alpha,
$$

and carrying capacity $K=1+(p-1) B /(\beta C-\alpha)$. 
Taking into account random perturbation to the disease transmission coefficient $\beta$, (1.1) becomes

$$
d Z=[(p-1) B Z+(\beta C-\alpha)(1-Z) Z] d t+\rho C(1-Z) Z d B(t)
$$

By using the Fokker-Planck equation method, Roberts and Saha in [1] investigated the asymptotic behavior of solutions of (1.3) when $R_{0}=(p B+\beta C) /(\alpha+\beta)>1$ (i.e., intrinsic growth rate $r>0)$. Recently, Ding et al. in [2] gave some results to deal with the case of $R_{0}=(p B+\beta C) /(\alpha+\beta)<1$; for example, they showed that the zero solution of (1.3) was asymptotically stable provided that the intensity of random perturbation is sufficiently small.

In the real world, at the same time, mortality rate due to disease has often been affected by some factors, such as age, seasons, and food supply. Thus, in order to describe epidemic more reasonable, we suppose that the increase of the death rate $\alpha$ is subjected by random disturbance also, and the model (1.3) becomes

$$
d Z=[(p-1) B Z+(\beta C-\alpha)(1-Z) Z] d t+\rho_{1} C(1-Z) Z d B_{1}(t)-\rho_{2}(1-Z) Z d B_{2}(t) .
$$

Here, $B_{1}(t)$ and $B_{2}(t)$ are independent standard Brownian motions; $\rho_{1}$ and $\rho_{2}$ are the intensity of the environmental disturbance. In this paper, for the new model (1.4) we carried out complete parameters analysis for $R_{0}>1$ (i.e., intrinsic growth rate $r>0$ ), $R_{0}=1$ (i.e., $r=0$ ), and $R_{0}<1$ (i.e., $r<0$ ), respectively. Our results are generalizations of the ones in $[1,2]$. Some interesting details about the system are revealed. For instance, we proved that when $R_{0}<1$, the zero solution of (1.4) is globally asymptotically stable, no matter how large the intensity of stochastic fluctuation is. Moreover, the explicit expectation and variance of steady solution of (1.4) are also given. Thus, the statistic properties of dynamics of (1.4) are completely clear.

The main argument used in this paper is the classical Fokker-Planck equation method. Of course, to deal with stochastic differential equations without linear growth condition, there are some other effective methods; we refer the reader to the papers [3-6].

\section{Nonnegative Solutions}

Following, we show that the solution of the SDE model (1.4) is global existent and unique.

Theorem 2.1. Assume that $B, \beta, \alpha$, and $C$ are positive real numbers. Then for any initial value $Z_{0}\left(0 \leq Z_{0}<1\right)$, there is a unique solution $Z(t)$ to (1.4) on $t \geq 0$ and $0 \leq Z(t)<1$ almost surely; moreover, the solution $Z(t)$ to (1.4) will remain in $\mathbb{R}^{+}$with probability 1 .

Proof. It is obvious that the coefficients of (1.4) are locally Lipschitz continuous; hence, for any initial value $Z_{0}\left(0<Z_{0}<1\right)$, (1.4) has a unique local solution $Z(t), t \in\left[0, \tau_{e}\right)$. Moreover, by Gardiner [7, page 132], we obtain that system with reflecting barrier; hence, $Z(t)<1$ almost surely for any initial value $Z_{0}\left(0<Z_{0}<1\right)$. And, 0 is the solution of model (1.4); hence, the solution $Z(t)$ to $(1.4)$ for any initial value $Z_{0}\left(0 \leq Z_{0}<1\right)$ will remain in $\mathbb{R}^{+}$with probability 1. The proof is complete.

Remark 2.2. To prove that $Z(t)<1$, Gong gives other effective method [8, page 371]. 
Remark 2.3. To prove that global existence, there is other effective method also, (cf. [3]).

\section{The Fokker-Planck Equation}

We rewrite (1.4) as

$$
d Z=F(Z) d t+G_{1}(Z) d B_{1}(t)+G_{2}(Z) d B_{2}(t)
$$

where

$$
\begin{gathered}
F(Z)=(p-1) B Z+(\beta C-\alpha)(1-Z) Z ; \\
G_{1}(Z)=\rho_{1} C(1-Z) Z ; \\
G_{2}(Z)=-\rho_{2}(1-Z) Z .
\end{gathered}
$$

By Gard [9, page 144], the Fokker-Planck equation of this model is

$$
\frac{d^{2}}{d Z^{2}}\left\{\left[G_{1}^{2}(Z)+G_{2}^{2}(Z)\right] p(Z)\right\}-2 \frac{d}{d Z}[F(Z) p(Z)]=0,
$$

where $p(Z)$ is the steady density function, due to system with reflecting barrier [7, page 129], thus $p(Z)$ is proportional to

$$
\left(\frac{Z}{1-Z}\right)^{2 r / \rho^{2}} \frac{1}{Z^{2}(1-Z)^{2}} \exp \left\{\frac{2(p-1) B}{\rho^{2}(1-Z)}\right\}
$$

where

$$
\rho^{2}=\rho_{1}^{2} C+\rho_{2}^{2}
$$

and the constant of proportionality is determined by $\int_{0}^{1} p(Z) d Z=1$.

By differentiating (3.4), it is clear that the extremum of $p(Z)$ is related to the zeros of quadratic function:

$$
-2 \rho^{2} Z^{2}+\left(3 \rho^{2}-r+(p-1) B\right) Z+r-\rho^{2} .
$$

\section{The Asymptotic Behaviour of Solution}

To continue, we will regard the $\rho^{2}=\rho_{1}^{2} C+\rho_{2}^{2}$ as a parameter to separately discuss the asymptotic behaviour of the solutions of model (1.4) in three cases: intrinsic growth rate $r>0, r<0$, and $r=0$, respectively. 


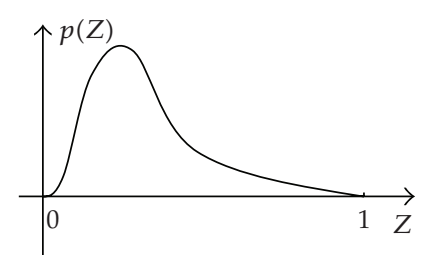

(a)

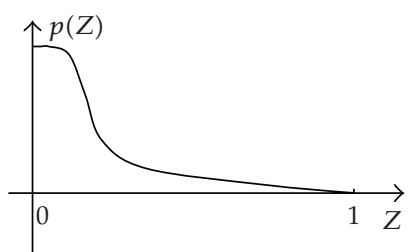

(b)

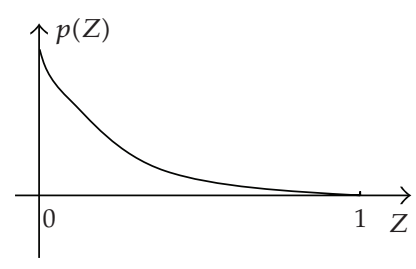

(c)

Figure 1

\subsection{Asymptotic Behaviour in the Case $r>0$}

Case $1(p \in[0,1))$. In this case, obviously, $\lim _{Z \rightarrow 1^{-}} p(Z)=0$.

(I) If $\rho^{2}<r$, then $\lim _{Z \rightarrow 0} p(Z)=0$. In (3.6), the function is negative when $Z=1$, positive when $Z=0$, and has unique root in $(0,1)$. Hence, the distribution $p(Z)$ tends to zero as $Z \rightarrow 0,1$ and is unimodal. The mode of $p(Z)$ to $K$ is $\rho \rightarrow 0$, and to zero is $\rho^{2} \rightarrow r$. (see, e.g., Figure 1(a).)

(II) If $\rho^{2}=r$, then $\lim _{Z \rightarrow 0} p(Z) \neq 0$. In (3.6), the function is negative when $Z=1$, and is zero when $Z=0$, and zero is unique root in $[0,1)$. Hence, the distribution $p(Z)$ is strictly monotonic decreasing function in $[0,1)$ with a positive maximum at $Z=0$ (see Figure 1(b).)

(III) If $r<\rho^{2}<2 r$ in (3.6), the function is negative when $Z=1$ and $Z=0$, and the $p(Z)$ is singular at $Z=0$ but integrable in $(0,1)$.

(i) If $3 \rho^{2}-2 \rho \sqrt{2\left(\rho^{2}-r\right)}<r-(p-1) B$, then function (3.6) is strictly negative for $0<Z<1$; hence the distribution $p(Z)$ is a monotonic decreasing function (see Figure 1(c).)

(ii) If $3 \rho^{2}-2 \rho \sqrt{2\left(\rho^{2}-r\right)}=r-(p-1) B$, then (3.6) has one zero for $0<Z<1$, the system occur a pitchfork bifurcation (see Figure 2(a).)

(iii) If $3 \rho^{2}-2 \rho \sqrt{2\left(\rho^{2}-r\right)}>r-(p-1) B$, then (3.6) has two zeros for $0<Z<1$, and the function $p(Z)$ is bimodal, with a singular peak at $Z=0$ and a positive maximum for some $0<Z<1$ (see Figure 2(b).)

(iv) If $\rho^{2} \geq 2 r$, then $p(Z)$ may be represented by a delta function at $Z=0$. That is, if perturbation sufficient is larger, the disease becomes extinct (see Figure 2(c).)

Case $2(p=1)$. (i) If $\rho^{2}<2 r$, then $p(Z)$ is a delta function at $Z=1$.

(ii) If $\rho^{2} \geq 2 r$, then $p(Z)$ consists of delta function at $Z=0$ and $Z=1$. The former corresponds to $\lim _{t \rightarrow \infty} Z(t)=1$ a.s., while for the later the limit for larger $t$ is either zero or one, the probability of each outcome being determined by the magnitudes of the residues of the density functions (3.3) at its extremes. 


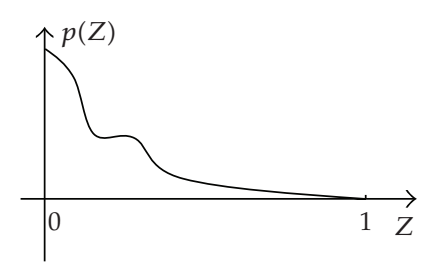

(a)

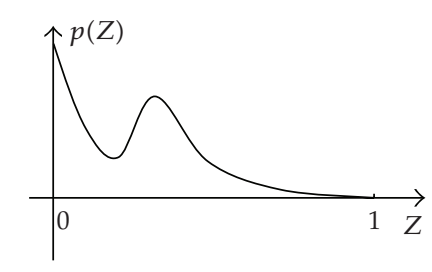

(b)

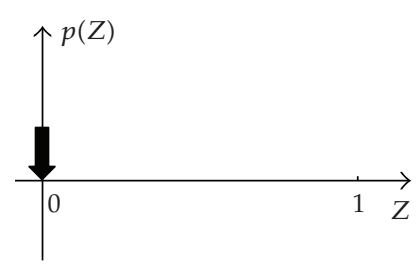

(c)

Figure 2

\subsection{Asymptotic Behaviour in the Case $r<0$}

Case $1(p \in[0,1))$. In this case, $\lim _{Z \rightarrow 1^{-}} p(Z)=0$. Moreover, for any $\rho$, obviously, $\rho^{2} \geq 2 r$, then $p(Z)$ may be represented by a delta function at $Z=0$. That is, for any perturbation, the disease becomes extinct; this is the same when there is no stochastic perturbation.

Case $2(p=1)$. In this case, $p(Z)$ consists of delta function at $Z=0$ and $Z=1$.

\subsection{Asymptotic Behaviour in the Case $r=0$}

Case $1(p \in[0,1))$. Equation (3.4) becomes

$$
\frac{1}{Z^{2}(1-Z)^{2}} \exp \left\{\frac{2(p-1) B}{\rho^{2}(1-Z)}\right\}
$$

and then $p(Z)$ may be represented by a delta function at $Z=0$.

Case $2(p=1)$. In this case, $p(Z)$ consists of delta function at $Z=0$ and $Z=1$.

\section{The Mean and Variance}

The first two moments of the distribution may be found directly from the differential equation by noting that $G_{1}(Z)=G_{2}(Z)=G_{1}^{\prime}(Z)=G_{2}^{\prime}(Z)=0$ if $Z=0$ or 1 ; from (3.3) we have

$$
\frac{d}{d Z}\left[\left(G_{1}^{2}(Z)+G_{2}^{2}(Z)\right) p(Z)\right]-2[F(Z) p(z)]=0 .
$$

Integrating again we obtain

$$
\int_{0}^{1} F(Z) p(Z) d Z=0
$$


or multiplying (5.1) by $Z^{-1}$ and integrating we obtain

$$
2 \int_{0}^{1} Z^{-1} F(Z) p(Z) d Z-\int_{0}^{1} Z^{-2}\left(G_{1}^{2}(Z)+G_{2}^{2}(Z)\right) p(Z) d Z=0
$$

Hence, solving (5.1) and (5.3), we obtain expression for the first moment and second moment of $Z$ :

$$
\begin{gathered}
E Z=\frac{\left(2 r-\rho^{2}\right)(\beta c-\alpha)}{\rho^{2} r+2(\beta c-\alpha)^{2}-2 \rho^{2}(\beta c-\alpha)} \\
E Z^{2}=\frac{r}{\beta c-\alpha} E Z .
\end{gathered}
$$

Hence, we obtain expression for the mean $\mu$ and variance $\sigma^{2}$

$$
\mu=E Z, \quad \sigma^{2}=E Z^{2}-\mu^{2}
$$

and embody

$$
\begin{gathered}
\mu=\frac{\left(2 r-\rho^{2}\right)(\beta c-\alpha)}{\rho^{2} r+2(\beta c-\alpha)^{2}-2 \rho^{2}(\beta c-\alpha)}, \\
\sigma^{2}=\frac{r\left(2 r-\rho^{2}\right)}{\rho^{2} r+2(\beta c-\alpha)^{2}-2 \rho^{2}(\beta c-\alpha)}-\left[\frac{\left(2 r-\rho^{2}\right)(\beta c-\alpha)}{\rho^{2} r+2(\beta c-\alpha)^{2}-2 \rho^{2}(\beta c-\alpha)}\right]^{2} .
\end{gathered}
$$

\section{Acknowledgments}

This research is supported by the National Science Foundation of China under Grants no. 10772140 and no. 10701020 and Harbin Institute Technology (Weihai) Science Foundation with no. HIT(WH)ZB200812.

\section{References}

[1] M. G. Roberts and A. K. Saha, "The asymptotic behaviour of a logistic epidemic model with stochastic disease transmission," Applied Mathematics Letters, vol. 12, no. 1, pp. 37-41, 1999.

[2] Y. Ding, M. Xu, and L. Hu, "Asymptotic behavior and stability of a stochastic model for AIDS transmission," Applied Mathematics and Computation, vol. 204, no. 1, pp. 99-108, 2008.

[3] X. Mao, G. Marion, and E. Renshaw, "Environmental Brownian noise suppresses explosions in population dynamics," Stochastic Processes and Their Applications, vol. 97, no. 1, pp. 95-110, 2002.

[4] J. He and K. Wang, "The survival analysis for a population in a polluted environment," Nonlinear Analysis: Real World Applications, vol. 10, no. 3, pp. 1555-1571, 2009.

[5] N. Dalal, D. Greenhalgh, and X. Mao, "A stochastic model for internal HIV dynamics," Journal of Mathematical Analysis and Applications, vol. 341, no. 2, pp. 1084-1101, 2008.

[6] D. Jiang, N. Shi, and X. Li, "Global stability and stochastic permanence of a non-autonomous logistic equation with random perturbation," Journal of Mathematical Analysis and Applications, vol. 340, no. 1, pp. 588-597, 2008. 
[7] C. W. Gardiner, Handbook of Stochastic Methods for Physics, Chemistry and the Natural Sciences, vol. 13 of Springer Series in Synergetics, Springer, Berlin, Germany, 3rd edition, 2004.

[8] G. Gong, Introduction to Stochastic Differential Equations, Beijing University Press, Beijing, China, 2nd edition, 2008.

[9] T. C. Gard, Introduction to Stochastic Differential Equations, vol. 114 of Monographs and Textbooks in Pure and Applied Mathematics, Marcel Dekker, New York, NY, USA, 1988. 


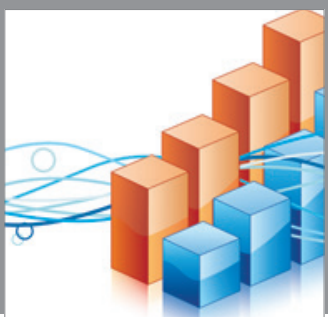

Advances in

Operations Research

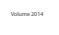

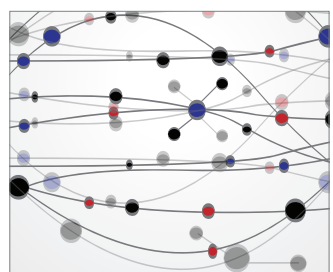

\section{The Scientific} World Journal
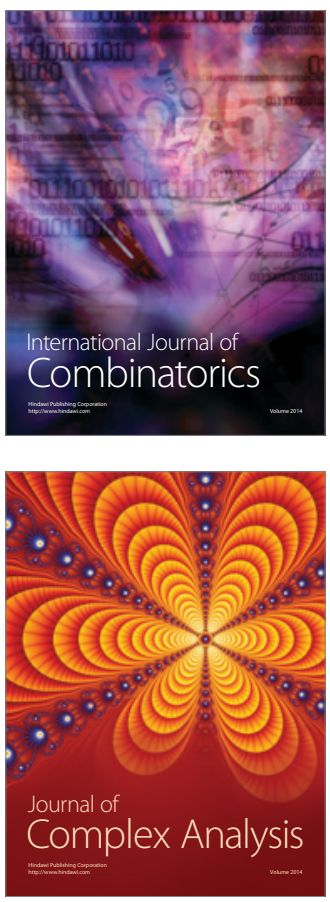

International Journal of

Mathematics and

Mathematical

Sciences
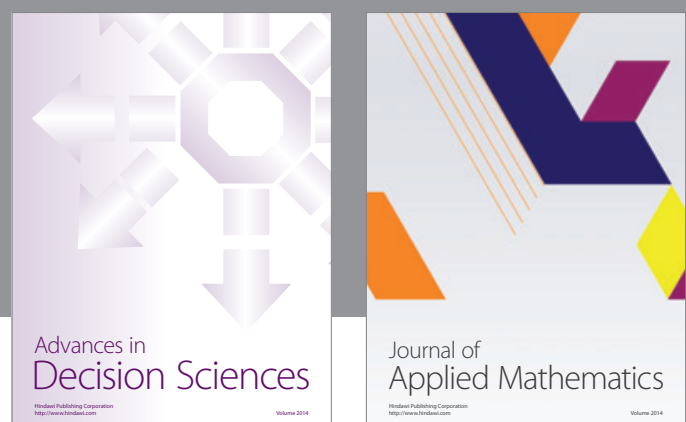

Journal of

Applied Mathematics
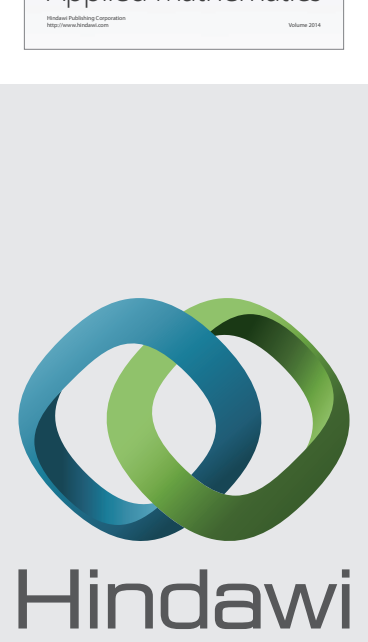

Submit your manuscripts at http://www.hindawi.com
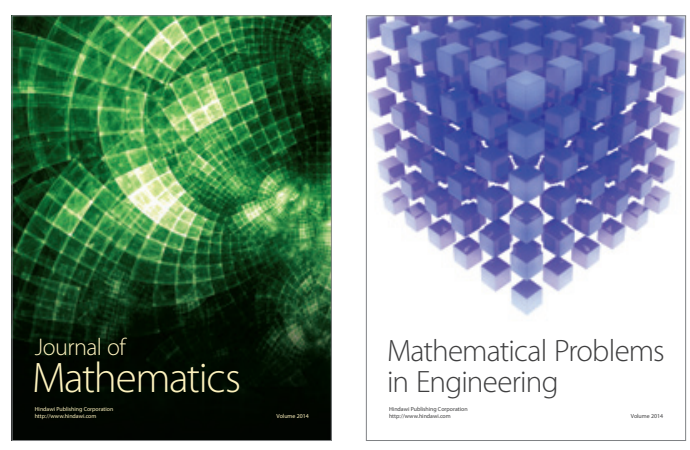

Mathematical Problems in Engineering
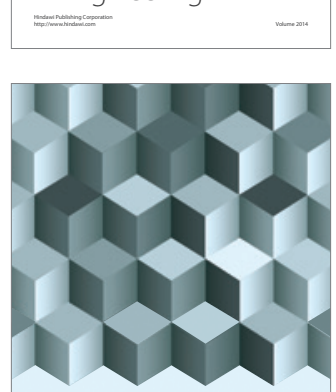

Journal of

Function Spaces
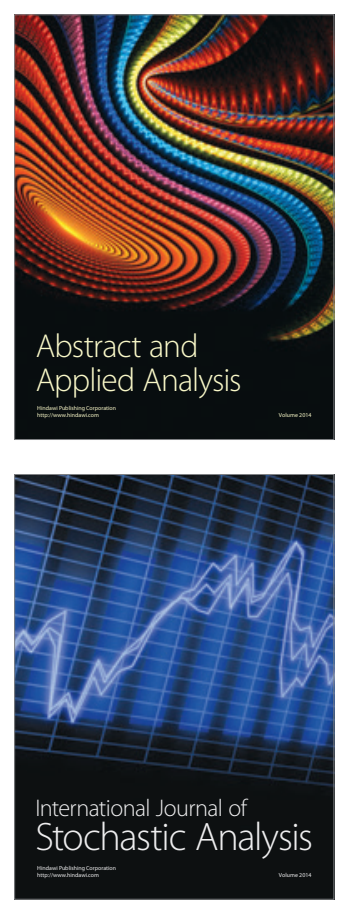

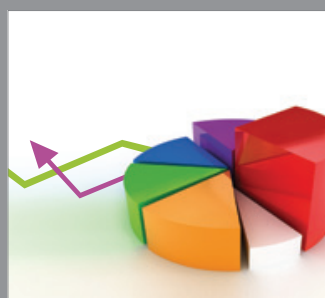

ournal of

Probability and Statistics

Promensencen
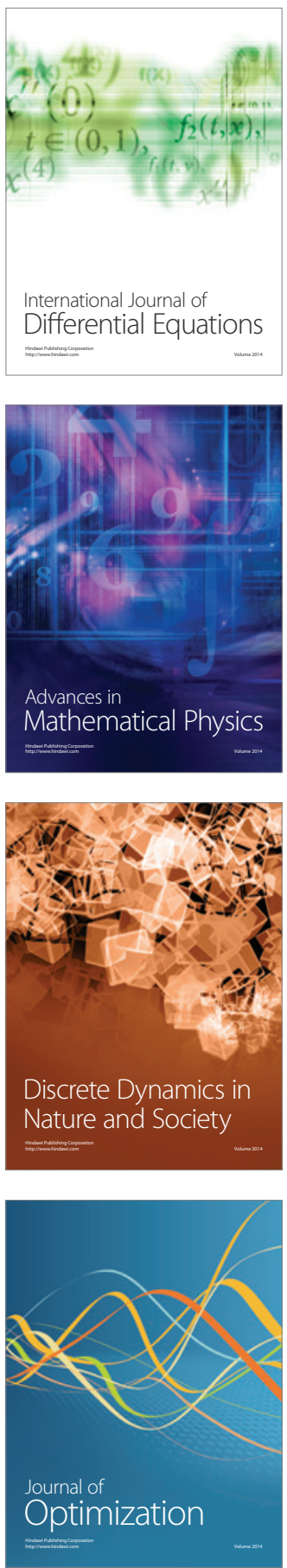